\title{
The Energy-Momentum Spectrum in Local Field Theories with Broken Lorentz-Symmetry
}

\author{
Hans-Jürgen Borchers ${ }^{1}$ and Detlev Buchholz ${ }^{2}$ \\ 1 Institut für Theoretische Physik der Universität Göttingen, D-3400 Göttingen, \\ Federal Republic of Germany \\ 2 II. Institut für Theoretische Physik der Universität Hamburg, D-2000 Hamburg, \\ Federal Republic of Germany
}

\begin{abstract}
Assuming locality of the observables and positivity of the energy it is shown that the joint spectrum of the energy-momentum operators has a Lorentz-invariant lower boundary in all superselection sectors. This result is of interest if the Lorentz-symmetry is (spontaneously) broken, such as in the charged sectors of quantum electrodynamics.
\end{abstract}

\section{Introduction}

The familiar theoretical explanation of the fact that the energy $p_{0}$ and the momentum $\mathbf{p}$ of an elementary particle are related by the fundamental equation $p_{0}$ $=\left(\mathbf{p}^{2}+m^{2}\right)^{1 / 2}, m$ being the mass of the particle, is based on the assumption that particle states can be described by vectors in some irreducible representation of the Poincaré group [1]. Alternatively, this form of the energy-momentum spectrum can be deduced from the hypothesis that the vectors describing particles can be generated from a vacuum vector with the help of local field operators [2]. The existence of Lorentz-transformations is not needed in the latter argument.

Both of these explanations are based on the (implicit) idea that, disregarding small "tails," particle states can be localized in bounded regions of space. Yet this picture is not correct in the case of particles carrying an electric charge. Since the electric charge is the source of the long-range Coulomb field, such states have poor localization properties which are incompatible with the existence of a continuous, unitary representation of the Lorentz group in the corresponding superselection sectors [3]. (Note that the geometrical action of a Lorentz transformation grows with the distance from the origin. So its effect on dislocalized states is large.) By the same token, there cannot exist local field operators generating these states from the vacuum [4]. Hence the conventional arguments establishing the Lorentzinvariance of the energy-momentum spectrum are not applicable in this case.

In the present article we reconsider the problem of the shape of the energymomentum spectrum in the general framework of local quantum theory [5]. This 
setting is appropriate for the discussion of theories with long range forces, such as quantum electrodynamics. We recall that the basic object in local quantum theory is the algebra of all local observables which, in the present analysis, may be regarded as a concrete ${ }^{*}$-algebra $\mathfrak{A}$ of (bounded) operators acting on the physical Hilbert space $\mathscr{H}$. As usual, the space-time translations $x \in \mathbb{R}^{4}$ are assumed to act as automorphisms $\alpha_{x}$ on $\mathfrak{U}$, but the existence of Lorentz-transformations is not required here.

Since the vectors in $\mathscr{H}$ are to describe elementary physical systems we assume that energy-momentum operators can be defined on $\mathscr{H}$ and that the energy is positive in all Lorentz-frames. This means that on $\mathscr{H}$ there exists a continuous, unitary representation $x \rightarrow U(x)$ of the translations implementing the automorphisms $\alpha_{x}$,

$$
U(x) A U(x)^{-1}=\alpha_{x}(A) \text { for } A \in \mathfrak{A},
$$

and the joint spectrum of the generators of $U$ is contained in the closed forward lightcone $\bar{V}$,

$$
\operatorname{sp} U \subset \bar{V} \text {. }
$$

It has been shown in [6] that under these conditions the operators $U(x)$ can be chosen from the weak closure of the observable algebra $\mathfrak{A}$,

$$
U(x) \in \mathfrak{U}^{-} .
$$

This fact is essential for the interpretation of the generators of $U$ as energymomentum operators. Yet since we do not rely on the existence of Lorentztransformations or fields generating the physical states from a vacuum vector, there exists a variety of representations $U$ of this kind: given any $p \in \bar{V}$ one can proceed from $U$ to the representation, ${ }^{1} x \rightarrow U^{\prime}(x)=U(x) \cdot e^{i p \cdot x}$, which also fulfills the conditions (1.1) to (1.3), but the spectra $\operatorname{sp} U$ and $\operatorname{sp} U^{\prime}$ are displaced by $p$. If $\mathscr{H}$ consists of several superselection sectors (i.e. if $\mathfrak{A}^{-}$has a non-trivial center) one can modify $U$ in this manner on each coherent subspace of $\mathscr{H}$, and this will in general change the spectrum substantially. So there arises the question as to which choice of the representation $U$ should be regarded as the most natural one.

We employ here the condition that a reasonable representation $U$ of the translations has to give rise to a minimal energy-operator. More precisely: if (with respect to a fixed Lorentz-frame) $H$ is the generator of the time-translations of $U$, and if $H^{\prime}$ is the corresponding generator of any other representation $U^{\prime}$ satisfying the conditions (1.1) $-(1.3)$, then

$$
H \leqq H^{\prime} \text {. }
$$

We will show in the subsequent section that such a minimal representation of the translations always exists. At the very end of our analysis it will also become clear that in local quantum theory this representation is unique.

Given these general constraints we will study in Sect. 3 which shape of the energy-momentum spectrum $\operatorname{sp} U$ is compatible with the principle of locality

1 If $a, b \in \mathbb{R}^{4}$ we denote by $a \cdot b$ the Lorentz-scalar product, which in proper coordinates is given by $a \cdot b=a_{0} \cdot b_{0}-\mathbf{a} \cdot \mathbf{b}$ 
(spacelike commutativity) of observables. It is essential for this part of our analysis that, according to this principle,

$$
\left[A^{*}, \alpha_{x}(A)\right]=0
$$

for all local operators $A \in \mathfrak{U}$ and space-like translations ${ }^{2} x \notin(\bar{V}-a) \cup(-\bar{V}+a)$, where $a$ is a sufficiently large time-like vector allowing for the finite localization of $A$. This property of the observables ties the translations $U$ to the causal structure of Minkowski space and thereby restricts the admissible shape of $\operatorname{sp} U$. Our main result can be stated as follows:

The lower boundary of $\operatorname{sp} U$ consists of the hyperboloid $\left\{p \in \mathbb{R}^{4}: p^{2}=m^{2}, p_{0} \geqq 0\right\}$ for some $m \geqq 0$ and, possibly, of the point $\{0\}$.

So also in the absence of Lorentz-transformations it turns out that the states at the bottom of the energy-momentum spectrum (amongst which one expects the elementary particles) satisfy the familiar relation between energy and momentum. This fact is of particular interest in quantum electrodynamics, where it adds to the understanding of the infra-particle problem as discussed in [7] (cf. also [8]). Another aspect of our results will be pointed out in Sect. 4.

Let us finally remark that, using locality, one can establish continuity properties of the spectral resolution of the spatial translations [9], just as in the presence of Lorentz-transformations $[10,11]$. So it seems plausible that the general invariance and continuity properties of the energy-momentum spectrum following from Lorentz-covariance are actually a consequence of the more fundamental principle of locality. Unfortunately, we have not yet been able to completely settle this point. But we believe that our methods can be improved so as to fill the remaining gaps. We hope to return to this problem in a future publication.

\section{Minimal Translations}

Let us now turn to the problem of exhibiting a representation $U$ of the translations which is minimal in the sense outlined in the introduction. To this end we fix a Lorentz-frame $\Sigma$ with time-direction given by a positive timelike vector $e$. This vector enters into the following

Definition. Let $U$ be a representation of the translations with the properties (1.1) (1.3). We say $U$ is $\Sigma$-minimal if the generator $H$ of the time-translations $\mathbb{R} \ni t$ $\rightarrow U(t \cdot e)$ is minimal, i.e. if the generator $H^{\prime}$ corresponding to any other choice $U^{\prime}$ of the translations satisfies $H^{\prime} \geqq H$. [Note that the generators of $U$ and $U^{\prime}$ commute because of condition (1.3). So there exists a joint core for $H$ and $H^{\prime}$.]

In the analysis of the subsequent section we will make use of another characterization of $\Sigma$-minimal translations which can be taken from the following

Lemma 2.1. Let $U$ be a representation of the translations satisfying the conditions (1.1)-(1.3). Then the following two statements are equivalent.

2 If $S, S^{\prime}$ are subsets of $\mathbb{R}^{4}$ we define $S \pm S^{\prime}=\left\{s \pm s^{\prime}: s \in S, s^{\prime} \in S^{\prime}\right\}$ 
i) $U$ is $\sum$-minimal

ii) There is no non-trivial subspace $\mathscr{H}^{\prime} \subset \mathscr{H}$ which is stable under the action of $\mathfrak{A}$ such that $^{3}$

$$
\operatorname{sp} U \uparrow \mathscr{H}^{\prime} \subset p+\bar{V}
$$

for some $p \in \mathbb{R}^{4}$ with $p \cdot e>0$.

We omit the simple proof of this equivalence.

For the proof that $\Sigma$-minimal translations always exist it is instructive to study first the case where the algebra $\mathfrak{A}$ acts irreducibly on $\mathscr{H}$. In this case it follows from condition (1.1) that any two representations $U$ and $U^{\prime}$ of the translations are related by

$$
U^{\prime}(x)=U(x) \cdot e^{-i p \cdot x}
$$

for some $p \in \mathbb{R}^{4}$. So given $U$ we must specify a $p$ such that $U^{\prime}$ is $\Sigma$-minimal. From the spectrum condition $\operatorname{sp} U^{\prime} \subset \bar{V}$ it follows that $p$ has to satisfy

$$
\operatorname{sp} U-p \subset \bar{V} \text {. }
$$

The requirement that the generator of the time-translations $t \rightarrow U^{\prime}(t \cdot e)$ is minimal amounts to the condition that $p \cdot e$ has the maximal value which is compatible with relation (2.2). That such vectors $p$ exist follows from the fact that the set of vectors $p^{\prime}$ satisfying (2.2) and $p^{\prime} \cdot e \geqq 0$ is compact and non-empty (since $\operatorname{sp} U \subset \bar{V}$ ). It should, however, be noticed that $p$ need not be unique since $\bar{V}$ is not a simplicial cone. This fact causes some problem of choice in the analysis of the general case, where $\mathfrak{A}$ is reducible 4 .

Turning now to this case, let $U$ be a fixed representation of the translations satisfying the conditions (1.1) $-(1.3)$. We denote by $\mathfrak{B}$ the set of all continuous, unitary representations $x \rightarrow W(x)$ of $\mathbb{R}^{4}$ with values in the center of $\mathfrak{A}^{-}$for which the translations $U^{\prime}$ given by

$$
U^{\prime}(x)=U(x) \cdot W(x)^{-1}
$$

fulfill again the spectrum condition. It is obvious that any $U^{\prime}$ satisfying the conditions (1.1) $-(1.3)$ can be represented in this way. What we want to show is that there is always a choice of $W \in \mathfrak{B}$ such that the resulting translations $U^{\prime}$ are $\sum$-minimal. To this end we divide $\mathfrak{B}$ into classes $\mathfrak{M}(K)$ of representations $W$ having the same generator $K$ for the subgroup $\mathbb{R} \cdot e \subset \mathbb{R}^{4}$. Between these classes we establish the following partial ordering:

$$
\mathfrak{W}\left(K^{\prime}\right) \succ \mathfrak{W}\left(K^{\prime \prime}\right) \quad \text { if } \quad K^{\prime} \geqq K^{\prime \prime} .
$$

We will show, using Zorn's lemma [12], that there exists a unique maximal element with respect to this ordering.

3 If $x \rightarrow V(x)$ is a representation of $\mathbb{R}^{4}$ on $\mathscr{H}$ and if $\mathscr{K} \subset \mathscr{H}$ is a subspace which is invariant under $V$ we denote by $V\lceil\mathscr{K}$ the restriction of $V$ to $\mathscr{K}$

4 The reader who is not interested in these technicalities may proceed directly to the subsequent section 
To begin with we note that every pair of classes $\mathfrak{M}\left(K^{\prime}\right), \mathfrak{M}\left(K^{\prime \prime}\right)$ has a joint upper bound. Namely, if $W^{\prime} \in \mathfrak{M}\left(K^{\prime}\right)$ and $W^{\prime \prime} \in \mathfrak{B}\left(K^{\prime \prime}\right)$, we can construct a representation $W$ setting

$$
W(x)=W^{\prime}(x) \cdot F+W^{\prime \prime}(x) \cdot(1-F),
$$

where $F$ is the projection onto the non-negative part of $K^{\prime}-K^{\prime \prime}$. It is obvious from relation (2.5) that $W \in \mathfrak{B}$. Moreover, the generator $K=K^{\prime} \cdot F+K^{\prime \prime} \cdot(1-F)$ of $t \rightarrow W(t \cdot e)$ is larger than $K^{\prime}$ and $K^{\prime \prime}$, respectively. Hence $\mathfrak{B}(K)$ is an upper bound for both, $\mathfrak{M}\left(K^{\prime}\right)$ and $\mathfrak{M}\left(K^{\prime \prime}\right)$. For the purpose of finding a maximal element we may therefore restrict our attention to the classes $\mathfrak{M}(K)>\mathfrak{M}(0)$.

Next, we analyse the spectrum of the representations $W \in \mathfrak{M}(K)$ on certain subspaces of $\mathscr{H}$. Let

$$
U(x)=\int e^{i p \cdot x} d E(p)
$$

be the spectral representation of the given translations and let $E(\Delta)$, where $\Delta \subset \mathbb{R}^{4}$ is any Borel set, be the corresponding spectral projections. We denote by $F_{n}, n \in \mathbb{N}$ the central support projection ${ }^{5}$ of $E_{n}=E(n \cdot e-\bar{V})$. Since $E_{n} \leqq E_{n^{\prime}}$ if $n \leqq n^{\prime}$ and $s \lim E_{n}=1$ we have clearly $F_{n} \leqq F_{n^{\prime}}$ for $n \leqq n^{\prime}$ and $s$-lim $F_{n}=1$. It is essential for our

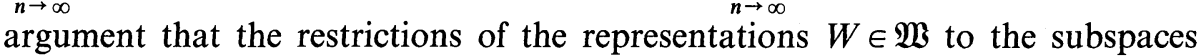
$F_{n} \cdot \mathscr{H}$ have their spectrum in $n \cdot e-\bar{V}$. This follows immediately from the inclusion

$$
\operatorname{sp} U\lceil F \mathscr{H} C \bar{V}+\mathrm{sp} W\lceil F \mathscr{H}
$$

(which holds for every non-zero central projection $F$ ) and the fact that $(n \cdot e-\bar{V}$ ) $\cap \operatorname{sp} U\left\lceil F_{n} \mathscr{H} \neq \emptyset\right.$. On the other hand, every representation $W \in \mathfrak{B}(K)>\mathfrak{B}(0)$ has its spectrum in the half space $\Sigma_{+}=\left\{k \in \mathbb{R}^{4}: k \cdot e \geqq 0\right\}$, hence

$$
\operatorname{sp} W \uparrow F_{n} \mathscr{H} \subset(n \cdot e-\bar{V}) \cap \Sigma_{+},
$$

and this set is compact.

We are now in a position to show that every linearly ordered set of classes $\mathfrak{W}\left(K_{\imath}\right)>\mathfrak{M}(0), \imath \in \mathbb{I}$ (II being some index set) has an upper bound. We pick from each class $\mathfrak{M}\left(K_{\imath}\right)$ a representation $W_{\imath}$ and consider the corresponding (four) generators $Q_{r}$. Since, according to relation (2.8), each representation $W_{l} \backslash F_{n} \mathscr{H}$ has its spectrum in the compact set $(n \cdot e-\bar{V}) \cap \Sigma_{+}$, it follows that the operators $Q_{\imath} \cdot F_{n}$ are uniformly bounded if $n$ is kept fixed. Hence, because of the weak compactness of the unit ball in a von Neumann algebra, the sequence $Q_{\imath} \cdot F_{n}, l \in \mathbb{I}$ contains a weakly convergent subnet. Its limit, which we denote by $Q^{(n)}$, is selfadjoint and bounded.

Now, according to the spectrum condition entering into the definition of $\mathfrak{B}$ we have for each $f \in \bar{V}$ the inequalities $P \cdot f \geqq Q_{\imath} \cdot f$ and $P \cdot f \geqq 0$, where $P$ is the generator of the translations $U$. Consequently $P \cdot f \geqq Q_{\imath} F_{n} \cdot f$ which gives in the above limit $P \cdot f \geqq Q^{(n)} \cdot f$. This means that the representation

$$
x \rightarrow W_{n}(x)=e^{i Q^{(n)} x}
$$

5 The central support projection $F$ of a projection $E \in \mathfrak{A}^{-}$is the smallest projection $F$ in the center of $\mathfrak{A}^{-}$such that $E \leqq F$ 
is an element of $\mathfrak{M}$. Setting $K^{(n)}=Q^{(n)} \cdot e$ and taking into account that the set $\mathfrak{M}\left(K_{\mathfrak{\imath}}\right)$, $\imath \in \mathbb{I I}$ is linearly ordered, we have furthermore

$$
K^{(n)} \geqq K_{l} \cdot F_{n} .
$$

To complete our argument we must proceed to the limit $n$ going to infinity. We do this as follows: let $G_{n}=F_{n}-F_{n-1}, n \in \mathbb{N}$ with the convention that $F_{0}=0$. Since $F_{n}$ $\geqq F_{n-1}$ and $\operatorname{s-lim}_{n \rightarrow \infty} F_{n}=1$, it is obvious that the operators $G_{n}$ are mutually orthogonal projections which sum up to 1 . So we can define a continuous, unitary representation $\bar{W}$ of $\mathbb{R}^{4}$, setting

$$
\bar{W}(x)=\sum_{n=1}^{\infty} W_{n}(x) \cdot G_{n} .
$$

Since $W_{n} \in \mathfrak{B}$ for each $n \in \mathbb{N}$ it is clear that $\bar{W} \in \mathfrak{B}$.

It remains to show that $\mathfrak{B}(\bar{K})$, where $\bar{K}$ is the generator of $t \rightarrow \bar{W}(t \cdot e)$, is an upper bound of $\mathfrak{M}\left(K_{\imath}\right), \imath \in \mathbb{I}$. According to the definition (2.11) we have $\bar{W} \uparrow G_{n} \mathscr{H}$ $=W_{n}\left\lceil G_{n} \mathscr{H}\right.$, so from relation (2.11) and the fact that $G_{n} \leqq F_{n}$ we obtain $\bar{K} G_{n} \geqq K_{\imath} G_{n}$. This holds for any $n \in \mathbb{N}$, and since $\sum_{n=1}^{\infty} G_{n}=1$ we arrive at the desired result $\bar{K} \geqq K_{l}$.

From the fact that every linearly ordered set of classes $\mathfrak{M}(K)$ has an upper bound it follows by Zorn's lemma that there is some maximal element $\mathfrak{B}\left(K_{0}\right)$. Moreover, since each pair of classes has a joint upper bound, it is also clear that $\mathfrak{M}\left(K_{0}\right) \succ \mathfrak{M}(K)$ for all $\mathfrak{M}(K)$, hence $\mathfrak{M}\left(K_{0}\right)$ is unique.

Now we pick any $W_{0} \in \mathfrak{B}\left(K_{0}\right)$ and define the translations

$$
x \rightarrow U_{0}(x)=U(x) \cdot W_{0}(x)^{-1} .
$$

It is then an immediate consequence of the definition of $\mathfrak{M}\left(K_{0}\right)$ that these translations are $\Sigma$-minimal. So we arrive at

Theorem 2.2. There exists a representation $x \rightarrow U_{0}(x)$ of the translations which is $\Sigma$-minimal.

As we already mentioned, the representation $x \rightarrow U_{0}(x)$ need not be unique since $\bar{V}$ is not a simplicial cone. This situation is, however, better in the cases where $\mathfrak{A}$ consists of local operators [in the sense of relation (1.5)]. Then one can proceed from condition (1.2) to the weaker assumption that $\operatorname{sp} U \subset \bar{V}_{s}$, where $\bar{V}_{s}$ is some simplicial cone whose faces are made up of characteristic planes (i.e. planes containing some lightlike vector). Since $\bar{V}_{s}$ is simplicial it follows from the spectral theory for automorphism groups (cf. for example [13]) that there exist unique minimal translations $U_{0}$ with $\operatorname{sp} U_{0} \subset \bar{V}_{s}$. Using locality and the special geometrical features of $\bar{V}_{s}$ one can argue then as in the subsequent section and show that actually $\operatorname{sp} U_{0} \subset \bar{V}$. So $\bar{V}$ is an admissible cone (cf. [13]) if $\mathfrak{A}$ consists of local operators.

The analysis of the present section, however, shows that no specific information on $\mathfrak{U}$ is needed if one only wants to select representation $U$ of the translations with a minimal energy-operator. 


\section{Locality and the Shape of the Spectrum}

Assuming from now on that the algebra $\mathfrak{A}$ consists of local operators [in the sense of relation (1.5)] we will analyze in this section which shape of the energymomentum spectrum is compatible with the principle of locality. To this end we fix a $\Sigma$-minimal representation $U$ of the translations and study the family of functions given in the following

Definition. Let $A \in \mathfrak{A}$ be any local operator and let $\Phi \in \mathscr{H}$. We consider the functions of $x \in \mathbb{R}^{4}$

$$
\begin{gathered}
\Delta_{A, \Phi}^{+}(x)=\left(\Phi, A^{*} U(x) A \Phi\right), \\
\Delta_{A, \Phi}^{-}(x)=\left(\Phi, \alpha_{x}(A) \cdot A^{*} U(x) \Phi\right),
\end{gathered}
$$

and the commutator function

$$
\Delta_{A, \Phi}(x)=\left(\Phi,\left[A^{*}, \alpha_{x}(A)\right] U(x) \Phi\right),
$$

which is the difference between $\Delta_{A, \Phi}^{+}$and $\Delta_{A, \Phi}^{-}$.

The functions $\Delta_{A, \Phi}^{+}$and $\Delta_{A, \Phi}^{-}$are continuous and uniformly bounded, so their Fourier-transforms $\tilde{U}_{A, \Phi}^{+}$and $\tilde{U}_{\boldsymbol{A}, \Phi}^{-}$, respectively, are defined as temperate distributions. Because of the spectrum condition (1.2) we have the following information on the supports of these distributions ${ }^{6}$ :

$$
\begin{gathered}
\operatorname{supp} \tilde{\Delta}_{A, \Phi}^{+} \subset \operatorname{sp} U \subset \bar{V}, \\
\operatorname{supp} \tilde{\Delta}_{A, \Phi}^{-} \subset(2 \cdot \operatorname{supp} \Phi-\operatorname{sp} U) \subset(2 \cdot \operatorname{supp} \Phi-\bar{V}),
\end{gathered}
$$

where $\operatorname{supp} \Phi$ denotes the support of the Fourier-transform of $x \rightarrow U(x) \Phi$. Consequently

$$
\operatorname{supp} \tilde{\Delta}_{A, \Phi} C \operatorname{sp} U \cup(2 \cdot \operatorname{supp} \Phi-\operatorname{sp} U) C \bar{V} \cup(2 \cdot \operatorname{supp} \Phi-\bar{V}) .
$$

The latter region has a non-trivial, open complement if $\operatorname{supp} \Phi$ is compact, which we will assume henceforth.

Using locality, it follows from the "edge of the wedge theorem" that $\tilde{\Delta}_{A, \Phi}$ is the discontinuity on the reals of some analytic function. This fact imposes restrictions on the possible shape of $\operatorname{supp} \tilde{\Delta}_{A, \Phi}$ which we will exploit later in our analysis of $\operatorname{sp} U$. For the convenience of the reader we recall here the essential steps leading to this result: the commutator function $\Delta_{A, \Phi}$ has, according to relation (1.5), support in the region $(\bar{V}-a) \cup(-\bar{V}+a)$, where $a \in \mathbb{R}^{4}$ depends on the localization properties of $A \in \mathfrak{U}$. Therefore one can decompose $\Delta_{A, \Phi}$ into the difference of two continuous functions $G_{+}$and $G_{-}$which have support in $(\bar{V}-a)$ and $(-\bar{V}+a)$, respectively. It follows from these support properties that the Fourier-transforms of $G_{ \pm}$are the boundary values (in the sense of distributions) of functions $H_{ \pm}$which are analytic in the tubes $T_{ \pm}=\left\{z \in \mathbb{C}^{4}: \operatorname{Im} z \in \pm V\right\}$, respectively. These boundary values coincide [because of relation (3.4)] in some open set $C \subset \mathbb{R}^{4}$. Thus $H_{+}$and $H_{-}$are, by the edge of the wedge theorem [14, Sect. 27], the restrictions to $T_{+}$and $T_{-}$, respectively, of a function $H$ which is analytic in $T_{+} \cup T_{-} \cup C^{\sim}$, where $C^{\sim}$ is

6 If $S$ is a subset of $\mathbb{R}^{4}$ we set $2 \cdot S=S+S$; cf. footnote 2 
some complex neighbourhood of $C$. Hence one obtains the basic relation

$$
\tilde{\Delta}_{A, \Phi}(k)=\lim _{\varepsilon}(H(k+i \varepsilon)-H(k-i \varepsilon))
$$

which holds on $\mathbb{R}^{4}$ (in the sense of distributions) for suitable sequences $\varepsilon \in V$ tending to 0 .

If one knows that $\tilde{\Delta}_{A, \Phi}$ vanishes in some region $C \subset \mathbb{R}^{4}$ it follows from relation (3.5) that $\tilde{\Delta}_{A, \Phi}$ also vanishes in the (in general larger) region $\hat{C}$ consisting of all real points of the envelope of holomorphy of the domain $T_{+} \cup T_{-} \cup C^{\sim}$. We will make use of this mechanism for various types of coincidence regions $C$, for which the completions $\hat{C}$ are explicitly known.

The first result, which is of a local character, is the "double cone theorem" [14, Sect. 28]. This theorem tells us that if the coincidence region $C$ contains an open neighbourhood of the timelike line $[0,1] \ni \lambda \rightarrow(1-\lambda) \cdot p_{1}+\lambda \cdot p_{2}$, where $p_{2} \in p_{1}$ $+\bar{V}$, then the completion $\hat{C}$ contains the double cone $\left(p_{1}+\bar{V}\right) \cap\left(p_{2}-\bar{V}\right)$. Actually, one can relax in this theorem the assumption that the points $p_{1}$ and $p_{2}$ are connected by a straight line. It suffices that $C$ contains a neighbourhood of some continuous curve $[0,1] \ni \lambda \rightarrow p(\lambda)$ with $p(0)=p_{1}, p(1)=p_{2}$, and $p(\lambda) \in p_{1}+\bar{V}$ for all $\lambda$. This generalized form of the double cone theorem follows by repeated application of the restricted version.

The second result, which is of a global nature, applies to coincidence regions $C$ which are the complement of regions of the form $\Gamma=\left(\Gamma_{1}+\bar{V}\right) \cup\left(\Gamma_{2}-\bar{V}\right)$, where $\Gamma_{1}$ and $\Gamma_{2}$ are closed subsets of $\bar{V}$. For such regions the envelope of holomorphy of $T_{+}$ $\cup T_{-} \cup C^{\sim}$ has been computed in [15]. It coincides with the one given by the Jost, Lehmann, Dyson representation [14, Sect. 32] cf. also [16]. Again it is possible to find the completion $\hat{C}$ of $C$ by elementary geometrical considerations: denoting by $H(a, \mu)$ the hyperboloids with apex $a \in \mathbb{R}^{4}$ and mass $\mu \geqq 0$,

$$
H(a, \mu)=\left\{k \in \mathbb{R}^{4}:(k-a)^{2}=\mu^{2}\right\},
$$

one must determine all sets $H(a, \mu)$ which do not intersect with $C$ and take their union

$$
\hat{\Gamma}=\bigcup\{H(a, \mu): H(a, \mu) \cap C=\emptyset\} .
$$

The complement of the closed set $\hat{\Gamma} \subset \mathbb{R}^{4}$ is then the desired completion $\hat{C}$. We will refer to this method of finding $\hat{C}$ as the "JLD-technique".

With this information on the support properties of $\widetilde{\Delta}_{A, \Phi}$ we can now turn to the analysis of $\operatorname{sp} U$. It follows from relation (3.3) that the support of $\tilde{\Delta}_{A, \Phi}^{+}$is a subset of $\operatorname{sp} U$ and that the distributions $\tilde{U}_{A, \Phi}^{+}(k)$ and $\tilde{U}_{A, \Phi}(k)$ coincide in the (open) complement of the region $(2 \cdot \operatorname{supp} \Phi-\bar{V})$. So the commutator functions $\widetilde{\Delta}_{A, \Phi}$, $A \in \mathfrak{A}$ can be used to study that part of the spectrum sp $U$ which does not lie in the region $(2 \cdot \operatorname{supp} \Phi-\bar{V})$. Thus there arises the question for which points $q \in \mathbb{R}^{4}$ one can find vectors $\Phi \neq 0$ such that $q \notin(2 \cdot \operatorname{supp} \Phi-\bar{V})$, i.e. $2 \cdot \operatorname{supp} \Phi \cap(q+\bar{V})=\emptyset$.

Definition. Let $q \in \mathbb{R}^{4}$. A vector $\Phi \in \mathscr{H}, \Phi \neq 0$ is said to be $q$-admissible if $\operatorname{supp} \Phi$ is compact and $2 \cdot \operatorname{supp} \Phi \cap(q+\bar{V})=\emptyset$. (Note that a $q$-admissible vector $\Phi$ is also $q^{\prime}-$ admissible for any $q^{\prime} \in q+\bar{V}$.) 
Using locality and the fact that $U$ is $\Sigma$-minimal, we will now show that there is a large supply of $q$-admissible vectors if $q \neq 0$ and $q \in \Sigma_{+}=\left\{k \in \mathbb{R}^{4}: k \cdot e \geqq 0\right\}$. In view of the above remark it suffices to establish this result for $q \in \Sigma_{0}=\left\{k \in \mathbb{R}^{4}: k \cdot e\right.$ $=0\}$.

Lemma 3.1. For every $q \in \Sigma_{+}, q \neq 0$ there exist $q$-admissible vectors $\Phi$. Moreover, if for a spectral projection $E(\Delta)$ (cf. relation (2.6)) corresponding to some Borel set $\Delta \subset \mathbb{R}^{4},\left(\Phi, A^{*} E(\Delta) A \Phi\right)=0$ for all $q$-admissible vectors $\Phi$ and local operators $A$, then $E(\Delta)=0$.

Proof. The proof of the existence of $q$-admissible vectors amounts to showing that there exists a $p \in \operatorname{sp} U$ such that $p \notin\left(\frac{1}{2} q+\bar{V}\right)$. Since $\left(\frac{1}{2} q+\bar{V}\right)$ is closed there exists then also a neighbourhood $\mathscr{N}_{p}$ of $p$ such that $2 \cdot \mathscr{N}_{p}$ is disjoint from $(q+\bar{V})$, hence any vector $\Phi$ with $\operatorname{supp} \Phi \subset \mathscr{N}_{p}$ is $q$-admissible.

Now if such a $p$ does not exist, then $\operatorname{sp} U \subset \bar{V} \cap\left(\frac{1}{2} q+\bar{V}\right)$, and it suffices to exclude this possibility for $q \in \Sigma_{0}, q \neq 0$. To this end we fix a compact, non-negligible set $\Gamma_{0}$ $C \operatorname{sp} U$ and pick a vector $\Psi$ with supp $\Psi \subset \Gamma_{0}$. Then we consider the commutator function $\tilde{\Delta}_{A, \Psi}$ which, for any local operator $A$, vanishes according to relation (3.4) in the complement of $\left(\bar{V} \cap\left(\frac{1}{2} q+\bar{V}\right)\right) \cup\left(2 \Gamma_{0}-\bar{V}\right)$. To this coincidence region we can apply the JLD-technique, the conclusion being that $\tilde{\Delta}_{A, \Psi}$ also vanishes in the complement of $\Gamma_{1}+\bar{V} \cup(-\bar{V})$, where $\Gamma_{1}=\bar{V} \cap\left(\frac{1}{2} q+\bar{V}\right) \cap\left(2 \Gamma_{0}-\bar{V}\right)$. Consequently, the positive part $\widetilde{\Delta}_{A, \Psi}^{+}$of the commutator function vanishes in the complement of $\left(\Gamma_{1}+\bar{V}\right) \cup \Gamma_{2}$, where $\Gamma_{2}=\left(2 \Gamma_{0}-\bar{V}\right) \cap \bar{V}$ is a compact set which swallows the overlap region of the supports of $\tilde{\Delta}_{A, \Psi}^{+}$and $\tilde{\Delta}_{A, \Psi}^{-}$. If one rewrites this result in terms of matrix elements of the spectral projections $E(\Delta)$ one gets

$$
(1-E(\Gamma)) \cdot A \Psi=0,
$$

if $\Gamma \supset\left(\left(\Gamma_{1}+\bar{V}\right) \cup \Gamma_{2}\right) \cap \operatorname{sp} U$. Now $\Gamma_{1}$ and $\Gamma_{2} \cap \operatorname{sp} U$ are compact subsets of $\bar{V} \cap\left(\frac{1}{2} q\right.$ $+\bar{V})$, and since for any spacelike vector $q \neq 0$ the region $\bar{V} \cap\left(\frac{1}{2} q+\bar{V}\right)$ is contained in the open cone $\left(\frac{1}{4} q+V\right)$, there exists an open neighbourhood $\mathscr{N}$ of 0 such that $\Gamma_{1}$ $\cup\left(\Gamma_{2} \cap \operatorname{sp} U\right)+\mathscr{N} \subset\left(\frac{1}{4} q+\bar{V}\right)$. Hence relation (3.8) holds in particular if $\Gamma$ is such that $\Gamma+\mathscr{N} \supset \frac{1}{4} q+\bar{V}$.

If $\mathfrak{A}$ is irreducible, then the set of vectors $A \Psi, A \in \mathfrak{A}$ is dense in $\mathscr{H}$. So it follows from (3.8) that $E(\Gamma)=1$ if $\Gamma+\mathscr{N} \supset \frac{1}{4} q+\bar{V}$, which implies that $\operatorname{sp} U+\mathscr{N} \subset \frac{1}{4} q+\bar{V}$. Since $q \in \Sigma_{0}$ and $\mathscr{N}$ contains negative timelike vectors this is a contradiction to the assumption that $U$ is $\Sigma$-minimal. So in this case there exists a $q$-admissible vector $\Phi$, and using the irreducibility of $\mathfrak{A}$ once more it is also clear that $E(\Delta)=0$ if $(\Phi$, $\left.A^{*} E(\Delta) A \Phi\right)=0$ for all $A \in \mathfrak{A}$.

In the general case we conclude from relation (3.8) (because it holds for all $\Psi$ with supp $\left.\Psi \subset \Gamma_{0}\right)$ that $(1-E(\Gamma)) \mathfrak{A}^{-} E\left(\Gamma_{0}\right)=0$. So the central support projection $Z$ of $E\left(\Gamma_{0}\right)$ is orthogonal to $(1-E(\Gamma))$. But the relation $E(\Gamma) \cdot Z=Z$ for $\Gamma+\mathscr{N} \subset \frac{1}{4} q$ $+\bar{V}$ implies that $\operatorname{sp} U\left\lceil Z \mathscr{H}+\mathscr{N} \subset \frac{1}{4} q+\bar{V}\right.$, so again we are led to a contradiction. This establishes the existence of $q$-admissible vectors $\Phi$ in the general case. Finally, if for all $q$-admissible vectors $\Phi$ and local operators $A\left(\Phi, A^{*} E(\Delta) A \Phi\right)=0$, then $E(\Delta) \mathfrak{A}^{-}\left(1-E\left(\frac{1}{2} q+\bar{V}\right)\right)=0$, hence $E\left(\frac{1}{2} q+\bar{V}\right) \cdot Z^{\prime}=Z^{\prime}$, where $Z^{\prime}$ is the central support projection of $E(\Delta)$. Now if $Z^{\prime} \neq 0$ this would mean that $\operatorname{sp} U\left\lceil Z^{\prime} \mathscr{H} \subset \bar{V} \cap\left(\frac{1}{2} q\right.\right.$ $+\bar{V})$, and applying the previous arguments it would follow that $\operatorname{sp} U\left\lceil Z^{\prime} \mathscr{H}+\mathscr{N}\right.$ 
$C \frac{1}{4} q+\bar{V}$. This is not possible if $U$ is $\Sigma$-minimal. So $Z^{\prime}=0$ and consequently $E(\Delta)$ $=0$.

The above result shows that for any $q \in \Sigma_{0}, q \neq 0$ there exist points in $\operatorname{sp} U$ which lie in ${ }^{7} \bar{V} \cap C(q+\bar{V})$. If the Euclidean length of $q$ is small, this region is a thin layer about a part of the surface $\partial \bar{V}$ of $\bar{V}$, so there exist (almost) lightlike points in $\operatorname{sp} U$. Next, we explore the structure of $\operatorname{sp} U$ in the neighbourhood of points which can be connected with the complement of $\bar{V}$ by a curve which does not intersect with $\operatorname{sp} U$.

Lemma 3.2. Let $q \in V$ be a point which can be connected with the complement of $\bar{V}$ by a continuous curve which does not intersect with $\operatorname{sp} U$. Then, either $(q-V) \cap \operatorname{sp} U=\emptyset$ or $(q-V) \cap \operatorname{sp} U=\{0\}$. In the latter case 0 is an isolated (atomic) point of $\operatorname{sp} U$.

Proof. Let $\mathscr{C}$ be the curve connecting $q$ with the complement of $\bar{V}$. Since $\mathscr{C} \cap \operatorname{sp} U=\emptyset$ and $\operatorname{sp} U$ is closed, there is a whole neighbourhood of $\mathscr{C}$ which does not intersect with $\operatorname{sp} U$. So by slightly modifying $\mathscr{C}$ (if necessary) we can always assume that $\mathscr{C}$ is smooth. Moreover, we can assume that the (first) point $l$ where $\mathscr{C}$ penetrates $\partial \bar{V}$ is different from 0 . Let $l_{\Sigma}$ be the projection of $l$ onto the spacelike plane $\Sigma_{0}$. Then we continue the curve from $l$ to the point $\varepsilon \cdot l_{\Sigma}, \varepsilon>0$ along a straight line. This section lies outside of $\bar{V}$, so it is clearly disjoint from $\operatorname{sp} U$. It is also obvious that the whole curve connecting $q$ and $\varepsilon \cdot l_{\Sigma}$ is contained in the cone $\varepsilon \cdot l_{\Sigma}$ $+\bar{V}$ if $\varepsilon$ is sufficiently small.

Next we fix such an $\varepsilon$ and pick a vector $\Phi$ which is $\varepsilon \cdot l_{\Sigma}$-admissible; such vectors exist according to the previous lemma since $\varepsilon \cdot l_{\Sigma} \in \Sigma_{0}$ and $\varepsilon \cdot l_{\Sigma} \neq 0$. The above geometrical preparations guarantee that the commutator functions $\tilde{\Delta}_{A, \Phi}$ vanish in a neighbourhood of the curve connecting $q$ and $\varepsilon \cdot l_{\Sigma}$. Moreover, this curve is contained in the cone $\varepsilon \cdot l_{\Sigma}+\bar{V}$. So we can apply the (generalized) double cone theorem, which says that $\tilde{\Delta}_{A, \Phi}$ vanishes in the double cone $(q-\bar{V}) \cap\left(\varepsilon \cdot l_{\Sigma}+\bar{V}\right)$. Since the vector $\Phi$ is $\varepsilon \cdot l_{\Sigma}$-admissible, $\tilde{\Delta}_{A, \Phi}^{+}$vanishes too in this region, i.e.

$$
\left(\Phi, A^{*} E\left((q-V) \cap\left(\varepsilon \cdot l_{\Sigma}+V\right)\right) A \Phi\right)=0 .
$$

This result is independent of the choice of the local operator $A$ and the $\varepsilon \cdot l_{\Sigma^{-}}$ admissible vector $\Phi$. So applying once more the previous lemma it follows that $E\left((q-V) \cap\left(\varepsilon \cdot l_{\Sigma}+V\right)\right)=0$, which means that there is no energy-momentum spectrum in the open set $(q-V) \cap\left(\varepsilon \cdot l_{\Sigma}+V\right)$. This shows, since $\varepsilon$ can be made arbitrarily small, that $\operatorname{sp} U$ is disjoint from $(q-V) \cap V$ as well as from the part of $(q-V) \cap \partial \bar{V}$ lying in the half-space $\left\{k \in \mathbb{R}^{4}: k \cdot l_{\Sigma}<0\right\}$.

For the analysis of the remaining part we note that one can replace in this statement $l$ by any $l^{\prime} \in(q-V) \cap \partial \bar{V} \cap\left\{k \in \mathbb{R}^{4}: k \cdot l_{\Sigma}<0\right\}$ because, as a consequence of the above result, any of these points can be connected with $q$ by a continuous curve in the complement of $\operatorname{sp} U$. Repeating this procedure, one obtains sufficiently many points $l^{\prime}$ so that the corresponding half-spaces $\left\{k \in \mathbb{R}^{4}: k \cdot l_{\Sigma}^{\prime}<0\right\}$ generate a covering of $\mathbb{R}^{4} \backslash\{0\}^{8}$. It is then clear that $\operatorname{sp} U$ and $(q-V) \cap \bar{V}$ can only have the point 0 in common, if any. If $(q-V) \cap \operatorname{sp} U=\{0\}$, then 0 is an isolated point of $\operatorname{sp} U$

7 If $S \subset \mathbb{R}^{4}$ we denote by $C S$ the complement of $S$ in $\mathbb{R}^{4}$

8 Note that this statement would not hold in two space-time dimensions 
since $(q-V)$ contains an open neighbourhood of 0 . So in this case 0 belongs to the atomic part of $\operatorname{sp} U$.

We are now in a position to determine the shape of the lower boundary of the energy-momentum spectrum. We begin with a formal

Definition. Let $S \subset \bar{V}$ be a closed set. A point $p \in S$ is said to be a lower boundary point of $S$ if in any neighbourhood of $p$ there exists a $q$ which can be connected with the complement of $\bar{V}$ by a continuous curve which does not intersect with $S$. The set of all lower boundary points of $S$ is called the lower boundary of $S$.

The preceding lemma implies that the lower boundary of $\operatorname{sp} U$ is a continuous surface (disregarding the point 0 in the cases where 0 is an isolated point of $\operatorname{sp} U$ ). To make this obvious let us parametrize the lower boundary of $\operatorname{sp} U$ by the points in a spacelike plane, given in proper coordinates by $\left\{\left(q_{0}, \mathbf{q}\right): q_{0}=0\right\}$. For any such point $(0, \mathbf{p})$ it follows from Lemma 3.2 that the timelike line $(\lambda, \mathbf{p}), \lambda \in \mathbb{R}$ has a nontrivial intersection with $\operatorname{sp} U$ (excluding the trivial case $\operatorname{sp} U=\{0\}$ ). On the other hand, $(\lambda, \mathbf{p}) \notin \operatorname{sp} U$ for negative $\lambda$, so we can define a mapping $\sigma:(0, \mathbf{p}) \rightarrow\left(p_{0}, \mathbf{p}\right)$ of the spacelike plane into the lower boundary of $\operatorname{sp} U$ setting

$$
p_{0}=\inf \{\lambda>0:(\lambda, \mathbf{p}) \in \operatorname{sp} U\} .
$$

This mapping is also onto (apart from the cases where 0 is an isolated point of $\operatorname{sp} U$ which then is not contained on the range of $\sigma$ ) since by Lemma 3.2 any two lower boundary points $p^{\prime} \neq 0$ and $p^{\prime \prime} \neq 0$ are spacelike or lightlike separated. From the latter fact it follows also that $\sigma$ is continuous and that its range is connected.

It is a simple but important consequence of this discussion that $\operatorname{sp}^{\prime} U$ (where the prime indicates that 0 has to be removed from the set $\operatorname{sp} U$ if it is an isolated point) and $\mathrm{sp}^{\prime} U+\bar{V}$ have the same lower boundary. We will exploit this fact in the proof of our main result.

Theorem 3.3. The lower boundary of $\mathrm{sp} U$ is a Lorentz invariant set. If $\mathfrak{A}$ is reducible, this statement holds also for $\mathrm{sp} U\left\lceil\mathscr{H}^{\prime}\right.$ whenever the subspace $\mathscr{H}^{\prime} \mathrm{C} \mathscr{H}$ is stable under the action of $\mathfrak{A}$. Moreover, $x \rightarrow U(x)$ is the only representation of the translations with this property (amongst the representations satisfying the conditions (1.1)-(1.3)).

Proof. We distinguish 3 cases corresponding to the possible shape of $\operatorname{sp} U$ at 0 .

i) $0 \notin \operatorname{sp} U$. Then there is a whole neighbourhood of 0 which does not belong to $\operatorname{sp} U$. Consequently, there exists some timelike vector $f \in V$ such that $\operatorname{sp} U \subset f+\Sigma_{+}$ and $\operatorname{sp} U \cap\left(f+\Sigma_{0}\right) \neq \emptyset$, i.e. the spacelike plane $f+\Sigma_{0}$ is tangent to $\operatorname{sp} U$. Now let $H(0, m), m \neq 0$ be the unique hyperboloid with apex 0 for which $f+\Sigma_{0}$ is a tangent plane, and let $p$ be the point where the hyperboloid and the plane intersect. We want to show that this hyperboloid is the lower boundary of $\operatorname{sp} U$. To this end we choose any $q \in \Sigma_{0}, q \neq 0$ and $\varepsilon>0$. Then we pick a vector $\Phi$ which is $\varepsilon \cdot q$-admissible and consider the commutator functions $\tilde{\Delta}_{A, \Phi}$. These functions vanish in the region

$$
C((\operatorname{sp} U+\bar{V}) \cup(2 \cdot \operatorname{supp} \Phi-\bar{V})),
$$

so that we can apply the JLD-technique. Now the hyperboloids $H(a, \mu)$ which do not enter this region are centered at points

$$
a \in \bar{V} \cap \complement(\varepsilon \cdot q+V),
$$


and satisfy

$$
H_{+}(a, \mu) \cap(\varepsilon \cdot q+\bar{V}) \subset \operatorname{sp} U+\bar{V}
$$

where $H_{+}(a, \mu)$ denotes the upper shell of $H(a, \mu)$. In both of these statements we have used the fact that $\Phi$ is $\varepsilon \cdot q$-admissible. Note, however, that in the conditions (3.12) and (3.13) the dependence of the hyperboloids on the specific choice of the support of $\Phi$ has been removed. So, irrespective of the choice of the local operator $A$ and the $\varepsilon \cdot q$-admissible vector $\Phi$, all commutator functions $\tilde{\Delta}_{A, \Phi}$ vanish in a neighbourhood of any point $k \in(\varepsilon \cdot q+\bar{V})$ which does not lie on one of the hyperboloids satisfying (3.12) and (3.13). Since for $k \in(\varepsilon \cdot q+V)$ we have $\tilde{\Delta}_{A, \Phi}(k)$ $=\tilde{\Delta}_{A, \Phi}^{+}(k)$, it follows then from Lemma 3.1 that $\operatorname{sp} U \cap(\varepsilon \cdot q+V)$ is contained in the union of these hyperboloids.

In order to simplify the subsequent geometrical discussion we proceed at this point to the limit $\varepsilon \rightarrow 0$. In this limit, the information obtained so far can be summarized in the inclusion

$$
\begin{gathered}
\operatorname{sp} U \cap V \subset \bigcup\left\{H_{+}(l, \mu): H_{+}(l, \mu) \subset \operatorname{sp} U+\bar{V},\right. \\
l \in \partial \bar{V}, l \cdot q \geqq 0, \mu \geqq 0\} .
\end{gathered}
$$

The apices $l$ and masses $\mu$ of the hyperboloids in (3.14) satisfy $(p-l)^{2} \leqq \mu^{2}$, where $p$ is the point of contact of $H(0, m)$ and $f+\Sigma_{0}$ : namely, either $l$ and $p$ are spacelike or lightlike separated, i.e. $(p-l)^{2} \leqq 0$, or $l$ lies below the plane $\left(f+\Sigma_{0}\right)$. In the latter case, the upper and lower shells of the hyperboloids in (3.14) are separated by $\left(f+\Sigma_{0}\right)$, and since $p$ is a point in this plane we get $(p-l)^{2} \leqq \mu^{2}$. Let us consider now the points $k$ on the hyperboloids in (3.14) which can be represented in the form

$$
k=p+\delta \cdot q+f^{\prime}
$$

for some $f^{\prime} \in \bar{V}$ and $\delta \geqq 0$. Since $(k-l)^{2}=\mu^{2} \geqq(p-l)^{2}, l \in \partial \bar{V}, l \cdot q \geqq 0$ and $p^{2}=m^{2}$ we get

$$
k^{2}-m^{2} \geqq 2 l \cdot(k-p)=2\left(\delta l \cdot q+l \cdot f^{\prime}\right) \geqq 0 .
$$

This shows that every point $k \in \operatorname{sp} U$ which can be represented in the form (3.15) satisfies $k^{2} \geqq m^{2}$. But the choice of $q \in \Sigma_{0}$ in our argument was completely arbitrary, and since any point in $\mathrm{sp} U$ can be represented in the form (3.15) with a suitable $q \in \Sigma_{0}$, it follows from (3.14) that $\operatorname{sp} U \cap V \subset H_{+}(0, m)+\bar{V}$. Consequently, there can be no spectrum on $\partial \bar{V}$ (otherwise the lower boundary of $\operatorname{sp} U$ would consist of disconnected parts, which is impossible), so we arrive at $\operatorname{sp} U C H_{+}(0, m)$ $+\bar{V}$. Moreover, $p$ must be an element of $\operatorname{sp} U$ because $p$ is the only point in $H_{+}(0, m)+\bar{V}$ which is also an element of the spacelike plane $f+\Sigma_{0}$, and by assumption $\operatorname{sp} U \cap\left(f+\Sigma_{0}\right) \neq \emptyset$.

In the final step of our argument we feed this information into relation (3.14): since $p \in \operatorname{sp} U$ there must be a hyperboloid $H(l, \mu)$ which contains $p$ and whose upper shell lies in $\operatorname{sp} U+\bar{V} C H_{+}(0, m)+\bar{V}$. It is easy to verify that the only hyperboloid with these properties is $H(0, m)$, so we have $H_{+}(0, m) \subset \operatorname{sp} U+\bar{V}$, and consequently $H_{+}(0, m)$ is the lower boundary of $\operatorname{sp} U+\bar{V}$. But the lower boundary of $\operatorname{sp} U+\bar{V}$ and $\operatorname{sp} U$ coincide, so the first part of the theorem follows if $0 \notin \operatorname{sp} U$. 
ii) $0 \in \operatorname{sp} U$ is an isolated point. Then one can argue as in the previous case, the only difference being that one must replace everywhere the set $\operatorname{sp} U$ by $\operatorname{sp}^{\prime} U$. Actually, one has much stronger results in this case: it has been shown in [2] that if $\mathscr{H}$ contains a vector $\Omega$ (vacuum) which is cyclic for $\mathfrak{A}$ and invariant under $U(x)$, then the whole spectrum $\operatorname{sp} U$ is a Lorentz-invariant set.

iii) $0 \in \operatorname{sp} U$ is not isolated. Then, according to Lemma 3.2, there does not exist any lower boundary point in the interior of $\bar{V}$, and consequently the lower boundary of $\operatorname{sp} U$ consists of $\partial \bar{V}$.

So $\operatorname{sp} U$ has in all these cases a Lorentz-invariant lower boundary. This is also true for sp $U\left\lceil\mathscr{H}^{\prime}\right.$ whenever the subspace $\mathscr{H}^{\prime} \subset \mathscr{H}$ is stable under $\mathfrak{A}$, since one can apply the previous argument to any subrepresentation of $\mathfrak{A}$.

Finally, let $x \rightarrow U_{1}(x) \in \mathfrak{U}^{-}$be another unitary representation inducing the translations on $\mathfrak{A}$ whose spectrum has a Lorentz-invariant lower boundary in every subrepresentation of $\mathfrak{A}$. From the fact that both, $U$ and $U_{1}$ implement the translations on $\mathfrak{A}$, it follows that $W(x)=U_{1}(x) U(x)^{-1}$ is an element of the center of $\mathfrak{A}^{-}$. So $x \rightarrow W(x)$ is a continuous, unitary representation of $\mathbb{R}^{4}$. We denote the spectral projections corresponding to $W$ by $F(\Delta)$ and consider the subrepresentation of $\mathfrak{A}$ on $\mathscr{H}^{\prime}=F(\Delta) \mathscr{H}$. Since $U_{1}(x)=U(x) W(x)$ we get, provided $\mathscr{H}^{\prime} \neq 0$,

$$
\operatorname{sp} U_{1} \uparrow \mathscr{H}^{\prime} C \Delta+\operatorname{sp} U \uparrow \mathscr{H}^{\prime} .
$$

If $\Delta$ is a neighbourhood of any point $q \notin(-\bar{V})$ this leads immediately to a contradiction to the assumption that $\operatorname{sp} U\left\lceil\mathscr{H}^{\prime}\right.$ and $\operatorname{sp} U_{1}\left\lceil\mathscr{H}^{\prime}\right.$ have a Lorentzinvariant lower boundary. So for such sets we get $F(\Delta)=0$. Now we interchange the rôle of $U$ and $U_{1}$, using $U(x)=U_{1}(x) W(x)^{-1}$. This leads to

$$
\operatorname{sp} U\left\lceil\mathscr{H}^{\prime} C-\Delta+\operatorname{sp} U_{1} \uparrow \mathscr{H}^{\prime} .\right.
$$

So $F(\Delta)$ also vanishes if $\Delta$ is a neighbourhood of any point $p \notin \bar{V}$, and combining both results we arrive at $F(\Delta)=0$ if $0 \notin \Delta$. This shows that $W(x)=1$ and completes the proof of the theorem.

In the derivation of the above results we profited from the explicit information on the envelope of holomorphy of domains of the type $T_{+} \cup T_{-} \cup C^{\sim}$, where $C$ is an order convex region which lies between the two shells of some hyperboloid $H(a, \mu)$ (the JLD-technique). As is clear from this discussion, an extension of our methods to the analysis of the full energy-momentum spectrum, i.e. the problem of the possible shape of "holes" in sp $U$, would require a (partial) knowledge of the domain of holomorphy for coincidence regions $C$ consisting of an order convex part $C_{1}$ of the form $C((p-\bar{V}) \cup(-p+\bar{V}))$ and a double cone $C_{2}$ which is disjoint (but not spacelike separated) from $C_{1}$. Similar geometrical situations have been discussed in [17] (cf. also the remark in [18, p. 170]), and it seems plausible that these results can be applied to the present problem so as to establish the Lorentz invariance of the full spectrum. However, the details have yet to be worked out.

Without any additional effort we can, however, say something about the structure of the spectrum above the lower boundary in the special cases, where a part of the lower boundary is isolated from the rest of the spectrum.

Definition. Let $S \subset \bar{V}$ be a closed set and let $p \in S$ be a lower boundary point. The lower boundary of $S$ is said to be isolated at $p$ if there exists a neighbourhood $\mathscr{N}_{p}$ of 
$p$ such that $\mathscr{N}_{p} \cap S$ contains only lower boundary points. The lower boundary of $S$ is said to be completely isolated if it is isolated at all of its points.

Theorem 3.4. If the lower boundary of $\mathrm{sp}^{\prime} U$ is isolated at some point $p$, then it is completely isolated. Moreover, if one removes from $\mathrm{sp}^{\prime} U$ the lower boundary, then the remaining set has again a Lorentz-invariant lower boundary.

Proof. According to the previous theorem the lower boundary of $\mathrm{sp}^{\prime} U$ consists of the hyperboloid $H(0, m), m^{2}=p^{2}$, since $p$ is a lower boundary point of $\operatorname{sp}^{\prime} U$. We remove from $\mathrm{sp}^{\prime} U$ its lower boundary and denote the closure of the resulting set by $\operatorname{sp}^{\prime \prime} U$. Since the boundary of $\operatorname{sp}^{\prime} U$ is isolated at $p$, there exists then an open neighborhood $\mathscr{N}_{p}$ of $p$ such that $\operatorname{sp}^{\prime \prime} U \cap \mathscr{N}_{p}=\emptyset$.

Next we remove from the positive part $\tilde{\Delta}_{A, \Phi}^{+}$of the commutator functions $\tilde{\Delta}_{A, \Phi}$ the contributions coming from "intermediate states" with support on the lower boundary of $\operatorname{sp}^{\prime} U$. To this end we multiply $\tilde{\Delta}_{A, \Phi}^{+}(k)$ by $k^{2}-m^{2}$. Since $d^{4} k \tilde{\Delta}_{A, \Phi}^{+}(k)$ is a measure, it is clear that the resulting distributions $\left(k^{2}-m^{2}\right) \tilde{\Delta}_{A, \Phi}^{+}(k)$ have support in $\operatorname{sp}^{\prime \prime} U$. On the other hand, the supports of all these distributions are dense in $\mathrm{sp}^{\prime \prime} U$. It is crucial now that multiplying the commutator functions $\tilde{\Delta}_{A, \Phi}(k)$ by the polynomial $\left(k^{2}-m^{2}\right)$ does not destroy their analytic properties [in the sense of relation (3.5)]. So one can apply the same arguments as in Lemma 3.2 and Theorem 3.3 in order to show that the lower boundary of $\operatorname{sp}^{\prime \prime} U$ is a Lorentzinvariant set. Since $\operatorname{sp}^{\prime \prime} U \cap \mathscr{N}_{p}=\emptyset$, it follows then that $H(0, m)$ is disjoint from $\mathrm{sp}^{\prime \prime} U$, so the lower boundary of $\mathrm{sp}^{\prime} U$ is completely isolated.

It needs no explanation that in the situation described in this theorem one is dealing with a representation space $\mathscr{H}$ of $\mathfrak{A}$ containing single particle states. A detailed analysis of these representations can be found in [9].

Let us remark in conclusion that our results hold in any number of space-time dimensions. In two dimensions, however, one finds in the analysis of Lemma 3.2 the additional possibility that $\mathrm{sp} U$ can have an isolated part, consisting of the right or left branch of $\partial \bar{V}$ (cf. footnote 8 ). This kind of a spectrum describes massless particles moving only in one direction. Using the above methods one can also handle these cases, so the statements of both theorems hold true without modifications in any number of space-time dimensions.

\section{Concluding Remarks}

We have seen in the present investigations that there exists a canonical choice of the representation $x \rightarrow U(x)$ of the translations which is distinguished by the fact that its spectrum has a Lorentz-invariant lower boundary in each superselection sector. This result is a consequence of the principle of locality and holds even if the Lorentz-transformations are broken. In view of the uniqueness of the representation $x \rightarrow U(x)$ it is natural to regard the corresponding generators $P$ as the (global) observables of energy and momentum.

It should be noticed, however, that the energy momentum operators $P$ can in general only be used to compare the energy-content of states in the same superselection sector (in spite of the fact that $P$ is uniquely defined on the whole 
physical Hilbert space $\mathscr{H})$. The energy-difference of states in different superselection sectors of $\mathscr{H}$ is a priori not defined in terms of the spectrum of $P$ (cf. the example given below). So there arises the question of how to compare energies in different sectors.

If all physical states are local excitations of a unique vacuum state, then the answer is well known: given any charged state one can add to it a compensating charge "behind the moon" such that the resulting state has total charge 0 . Because of the large separation of the charges it then follows that the total energy of this state (in terms of $P$ ) is just the sum of the energies of the two constituents. This "additivity of the energy" (cf. for example [2]) makes it possible to relate the energies in different sectors so that the spectrum of $P$ gains an absolute meaning. This result can also be established without any a priori assumptions on the localization properties of the physical states if the particle spectrum of the theory is completely massive [9].

The situation is, however, less clear in theories with long-range forces and massless particles. The states in these models have in general poor localization properties, and, as a matter of fact, one can often distinguish the various superselection sectors by the different behaviour of the corresponding states at spacelike infinity (cf. for example [8]). So the heuristic ideas which led to a proof of the additivity of the energy in massive theories cannot readily be applied to the massless case, and $P$ need not always have an absolute meaning.

Yet since we are only interested in comparing energies in different sectors we could be content with less information. It would be sufficient to know how much energy is needed to proceed from one superselection sector to another. In our setting this quantity can be defined as follows: let $\mathscr{H}_{1}$ and $\mathscr{H}_{2}$ be arbitrary coherent subspaces of $\mathscr{H}$ (superselection sectors). It is then a very general fact (Fell's theorem ${ }^{9}$, cf. [5]) that any state $\Psi \in \mathscr{H}_{2}$ can be approximated by states in $\mathscr{H}_{1}$ in the weak topology, i.e. there exists a sequence (or, more generally, a net) $\Phi_{\imath} \in \mathscr{H}_{1}$ such that

$$
\lim _{\imath}\left(\Phi_{\imath}, A \Phi_{\imath}\right)=(\Psi, A \Psi) \quad \text { for } \quad A \in \mathfrak{A} .
$$

Now let $\Psi \in \mathscr{H}_{2}$ be a vector with energy-momentum about the lowest lying point $\left(m_{2}, \mathbf{0}\right)$ in $\operatorname{sp} U\left\lceil\mathscr{H}_{2}\right.$. Then we can ask whether it is possible to choose a sequence $\Phi_{t}$ in relation (4.1) with finite maximal energy $m_{1}$, i.e.

$$
\Phi_{\imath} \in E(\Delta) \mathscr{H}_{1} \quad \text { where } \quad \Delta=\left(\left(m_{1}, \mathbf{0}\right)-\bar{V}\right) \cap \bar{V} .
$$

If such an $m_{1}$ exists we say the sector $\mathscr{H}_{2}$ is energy-connected with $\mathscr{H}_{1}$; the energy which is necessary in order to proceed from $\mathscr{H}_{1}$ to $\mathscr{H}_{2}$ is then given by inf $\left(m_{1}-m_{2}\right)$. It is a simple consequence of our results on the shape of the energy-momentum spectrum that this quantity is always non-negative.

In this context there arises the following interesting question: does the property of energy-connectedness define an equivalence relation between superselection sectors? And if so, are the energies which are needed in order to proceed from $\mathscr{H}_{1}$ to $\mathscr{H}_{2}$ and from $\mathscr{H}_{2}$ to $\mathscr{H}_{1}$, respectively, equal? Both questions

9 We refrain from giving the weakest conditions under which Fell's theorem holds. For our purposes it is sufficient (and physically reasonable) to assume that $\mathfrak{A}$ is a simple $C^{*}$-algebra 
have clearly an affirmative answer in theories of massive particles. There the energy which is needed in order to proceed from one sector to another is the same for both directions, because to each (compensating) charge there exists, by the PCTtheorem, a conjugate charge with the same mass.

Let us finally discuss the significance of states lying in energy-disconnected sectors. An experimenter, who would try to prepare such a state in larger and larger space-time regions $\mathcal{O}$ would realize that this requires unlimited energy, although the energy inside the region $\mathcal{O}$ stays finite. So the energy which is needed for the preparation of this state is essentially a surface energy.

The simplest model in which these circumstances occur is the field-theory of a free, massless, scalar particle. In this model there exists a one-parameter family $\Omega_{\lambda}$, $\lambda \in \mathbb{R}$ of decent vacuum states [19] and it is straightforward to verify that the various vacuum sectors are energy-disconnected ${ }^{10}$. Note, however, that all vacuum states $\Omega_{\lambda}$ are eigenstates of the energy-momentum operator $P$ corresponding to the eigenvalue 0 ; so $P$ does not have a global meaning in this model.

It is not known whether the existence of energy-disconnected sectors in a model is always related to the existence of several vacuum states.

Acknowledgements. We are grateful to J. Bros for communicating to us his results on the analytic completion problem mentioned at the end of Sect. 3. One of us (D.B.) would also like to thank S. Doplicher, Universitá di Roma and A. S. Wightman, Princeton University for the warm hospitality extended to him during the early and final stages of this work. He also acknowledges financial support from the CNR, DFG, and Princeton University.

\section{References}

1. Wigner, E.P.: On unitary representations of the inhomogeneous Lorentz group. Ann. Math. 40, 149 (1939)

2. Borchers, H.J.: Local rings and the connection of spin with statistics. Commun. Math. Phys. 1, 281 (1965)

3. Fröhlich, J., Morchio, G., Strocchi, F.: Infrared problem and spontaneous breaking of the Lorentz group in QED. Phys. Lett. 89 B, 61 (1979)

4. Strocchi, F., Wightman, A.S.: Proof of the charge superselection rule in local relativistic quantum field theory. J. Math. Phys. 15, 2198 (1974)

5. Haag, R., Kastler, D.: An algebraic approach to quantum field theory. J. Math. Phys. 5, 848 (1964)

6. Borchers, H.J.: Translation group and spectrum condition. Commun. Math. Phys. 96, 1-13 (1984)

7. Fröhlich, J., Morchio, G., Strocchi, F.: Charged sectors and scattering states in quantum electrodynamics. Ann. Phys. 119, 241 (1979)

8. Buchholz, D.: The physical state space of quantum electrodynamics. Commun. Math. Phys. 85, 49 (1982)

9. Buchholz, D., Fredenhagen, K.: Locality and the structure of particle states. Commun. Math. Phys. 84, 1 (1982)

10. Hepp, K., Jost, R.: Über die Matrixelemente des Translationsoperators. Helv. Phys. Acta 35, 34 (1962)

11. Maison, D.: Eine Bemerkung zur Clustereigenschaft. Commun. Math. Phys. 10, 48 (1968)

12. Yosida, K.: Functional analysis. Berlin, Heidelberg, New York: Springer 1966

10 This result holds in four space-time dimensions. It is noteworthy that in three dimensions the corresponding sectors are energy-connected 
13. Pedersen, G.K.: $C^{*}$-algebras and their automorphism groups. London, New York, San Francisco: Academic Press 1979

14. Vladimirov, V.S.: Methods of the theory of functions of many complex variables. Cambridge, MA, London: MIT-Press 1966

15. Bros, J., Messiah, A., Stora, R.: A problem of analytic completion related to the JostLehmann-Dyson formula. J. Math. Phys. 2, 639 (1961)

16. Pflug, P.: Eine Bemerkung über die Konstruktion von Holomorphiehüllen. Zeszyty Nankowe Uniwersytetu Jagiellonskiego, Vol. 23

17. Borchers, H.J., Stora, R.: Unpublished notes (1963)

18. Stora, R.: Hyperfunctions and Theoretical Physics, Nice 1973. In: Lecture Notes in Mathematics, Vol. 449. Berlin, Heidelberg, New York: Springer 1975

19. Doplicher, S.: Fock representation and massless particles. Commun. Math. Phys. 3, 228 (1966)

Communicated by G. Mack

Received May 18, 1984 
